\title{
Towards the Optimum Light Source Spectrum
}

\author{
Andrew Chalmers and Snjezana Soltic \\ EET School Manukau Institute of Technology, Private Bag 94-006, Manukau 2241, New Zealand \\ Correspondence should be addressed to Andrew Chalmers, chalmers@manukau.ac.nz
}

Received 23 December 2009; Revised 24 April 2010; Accepted 7 June 2010

Academic Editor: Xian Cao

Copyright ( 12010 A. Chalmers and S. Soltic. This is an open access article distributed under the Creative Commons Attribution License, which permits unrestricted use, distribution, and reproduction in any medium, provided the original work is properly cited.

\begin{abstract}
This paper is concerned with designing light source spectra for optimum luminous efficacy and colour rendering. We demonstrate that it is possible to design light sources that can provide both good colour rendering and high luminous efficacy by combining the outputs of a number of narrowband spectral constituents. Also, the achievable results depend on the numbers and wavelengths of the different spectral bands utilized in the mixture. Practical realization of these concepts has been demonstrated in this pilot study which combines a number of simulations with tests using real LEDs (light emitting diodes). Such sources are capable of providing highly efficient lighting systems with good energy conservation potential. Further research is underway to investigate the practicalities of our proposals in relation to large-scale light source production.
\end{abstract}

\section{Introduction}

It is an aim of lighting source designers to produce sources that deliver effective light output at high efficiency combined with good colour rendering (the ability of a light source to display surfaces in their natural colours). Such aims are usually expressed in codes of practice (see, e.g. [1] for a review of the philosophy underlying lighting codes). With the prevailing emphasis on energy conservation, light sources are now also expected to have the highest practical luminous efficacy, for example, [2].

Since both colour rendering and luminous efficacy depend on the emitted spectrum of the source, there is considerable codependence between these properties. In fact, for the majority of "near-white" sources, these two parameters are generally contravariant. Good examples of this property can be found among the earlier (halophosphate type) families of fluorescent tubes, in which good colour rendering was associated with low luminous efficacy, and vice versa [3].

More recent fluorescent lamp designs have tended to exploit combinations of phosphors with narrow-band spectra at suitable wavelengths-providing enhanced luminous efficacy together with good colour rendering (e.g., [4]) breaking the above-mentioned "rule of thumb". Our research attempts to emulate this approach, to apply it to the design of mixed LED sources, and in the future to extend it to a more fundamental level - to the generalized design of optimized light sources.

This paper reports on a series of exploratory investigations during which the authors have gained an appreciation of the parameters of the questions under investigation, as part of the process of planning for specific future light source designs. It will also be demonstrated that mixed-LED white light sources could well become the optimum choice for high-colour rendering task lighting in the short to medium term.

\section{Assessment of Colour Rendering Performance}

For the past decade (and more) this has been a controversial issue within both the global lighting industry and the CIE (International Commission on Illumination) which produces standards and procedures for use by the industry. For the time being, the agreed definition of colour rendering performance is in terms of the CIE Colour Rendering Index [5]. This method suffers from certain shortcomings $[6,7]$ which are under investigation by CIE technical committee 
Table 1: The 14 CIE Test Colours [5].

\begin{tabular}{lcc}
\hline No. & Approximate Munsell Notation & Colour Appearance Under Daylight \\
\hline 1 & $7.5 \mathrm{R} 6 / 4$ & Light greyish red \\
2 & 5 Y $6 / 4$ & Dark greyish yellow \\
3 & $5 \mathrm{GY} 6 / 8$ & Strong yellow green \\
4 & $2.5 \mathrm{G} 6 / 6$ & Moderate yellowish green \\
5 & $10 \mathrm{BG} 6 / 4$ & Light bluish green \\
6 & $5 \mathrm{~PB} 6 / 8$ & Light Blue \\
7 & $2.5 \mathrm{P} 6 / 8$ & Light violet \\
8 & $10 \mathrm{P} 6 / 8$ & Light reddish purple \\
9 & $4.5 \mathrm{R} 4 / 13$ & Strong red \\
10 & $5 \mathrm{Y} 8 / 10$ & Strong yellow \\
11 & $4.5 \mathrm{G} 5 / 8$ & Strong green \\
12 & $3 \mathrm{~PB} 3 / 11$ & Strong blue \\
13 & $5 \mathrm{YR} 8 / 4$ & Light yellowish pink (light human complexion) \\
14 & $5 \mathrm{GY} 4 / 4$ & Moderate olive green (leaf green) \\
\hline
\end{tabular}

TC1-69 (Colour rendering of white light sources). For pragmatic reasons it has been decided to use the existing CIE method in the present investigation-and a brief summary of its main features is given below.

2.1. Method of CRI Calculation. The current CIE recommendation for calculating CRI (colour-rendering index) is described in CIE Publication 13.3 [5] which provides a standardised method for objective comparisons of the colour rendering properties of light sources. This method calculates the colour change of 14 test colours under the test source as compared with their colours under a reference source of the same CCT (correlated colour temperature) [8]. The reference illuminants are defined to be the CIE D-series of illuminants for CCT $\geq 5000 \mathrm{~K}$, and Planckian radiators for CCT $<5000 \mathrm{~K}$ [9]. The reference illuminant is chosen such that it has the same CCT as the test source. Residual differences in colour between the test source and reference illuminant are accounted for by using a von Kries type chromatic adaptation transform [10].

CCT is symbolized in this paper as $T_{C}$. Its significance is that it describes the chromaticity of the source, close to the Planckian locus in the CIE $(u, v)$ chromaticity diagram [11].

The fourteen test colours for this method are listed in Table 1. The CRI calculation is based on the CIE 1964 [12] colour difference formula.

$$
\Delta E_{i}=\sqrt{\left(U_{i}^{*}-U_{i o}^{*}\right)^{2}+\left(V_{i}^{*}-V_{i o}^{*}\right)^{2}+\left(W_{i}^{*}-W_{i o}^{*}\right)^{2}} .
$$

The terms containing $U^{*}$ and $V^{*}$ components represent the chromatic differences, and the $W^{*}$ term represents the lightness difference. Subscript $i$ refers to the test colour (sample) number, and subscript $o$ signifies the measure corresponding to the reference source.

Each test colour sample is assigned an individual ("special") colour rendering index:

$$
R_{i}=100-4.6 \Delta E_{i}
$$

The maximum value for $R_{i}$ is 100 -achievable only when the sample colour is unchanged under the test and reference sources. The coefficient of 4.6 for the subtractive term was chosen at the time the system was designed, such that $R_{a}$ (Equation (3) below) will have a value of $\sim 50$ when the test source is a "standard" warm white halophosphate-type fluorescent lamp of the type in use during the 1960s.

The general colour rendering index $R_{a}$ is defined as the average of the $R_{i}$ values for the first eight test colours:

$$
R_{a}=\frac{1}{8} \sum_{i=1}^{8} R_{i} .
$$

In some of the work described later in this paper, it was deemed expedient to use additional indices, based on the use of the six supplementary colour samples (Test Colours 9-14) and calculated by similar means to those for $R_{a}$ [1315]. These are referred to as $R_{b}$ and $R_{c}$, defined in (4). The justification for the use of these additional indices was that, under certain conditions, they appear to correlate better with visual experience than does the "official" index $R_{a}$,

$$
\begin{aligned}
& R_{b}=\frac{1}{6} \sum_{i=9}^{14} R_{i}, \\
& R_{c}=\frac{1}{14} \sum_{i=1}^{14} R_{i} .
\end{aligned}
$$

In addition, we have found it useful to be able to identify the test colour yielding the worst colour-rendering performance, by quoting its individual index $R_{\min }$ as well as the test colour number $i$ giving this minimum [16].

It is evident that $R_{i}$ can be negative (if $\Delta E_{i} \geq 21.7$ colorimetric units), and this was indeed found in certain instances, with some combinations of test sources and test colours. In extreme cases of poor colour rendering (where very large colour shifts are observed) it is also possible that the averaged indices, $R_{a}, R_{b}, R_{c}$ become negative.

In the remainder of this paper we use the abbreviation CRI to represent any one or more of the set: $R_{a}, R_{b}, R_{c}$. 


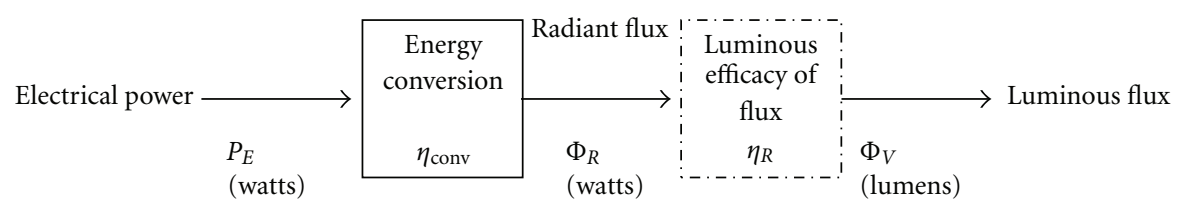

Figure 1: Lamp mechanisms.

\section{Light Source Efficiency and Efficacy}

The lamp may be considered to combine two mechanisms: an electrical-to-radiant energy conversion, together with a conceptual radiant-to-luminous flux conversion as shown in Figure 1. From this it is seen that the conversion efficiency is

$$
\left.\eta_{\text {conv }}=\frac{\Phi_{R}}{P_{E}} \text { (dimensionless }\right),
$$

and the radiant luminous efficacy is

$$
\eta_{R}=\frac{\Phi_{V}}{\Phi_{R}}\left(\frac{\operatorname{lm}}{\mathrm{W}}\right) .
$$

It should be noted that $\Phi_{V}$ is computed by making use of the $V_{\lambda}$ "standard visibility" weighting function [17].

The most commonly quoted lamp performance figure is the (overall) luminous efficacy of the lamp:

$$
\eta_{\text {lum }}=\frac{\Phi_{V}}{P_{E}}\left(\frac{\operatorname{lm}}{\mathrm{W}}\right) .
$$

Clearly,

$$
\eta_{\text {lum }}=\left(\frac{\Phi_{V}}{\Phi_{R}}\right) \times\left(\frac{\Phi_{R}}{P_{E}}\right)=\eta_{R} \times \eta_{\text {conv }} .
$$

However, since this paper is concerned primarily with the assessment of different light-source spectra, and not with the physical processes for producing those spectra, the focus of the present investigation is the radiant luminous efficacy (or, luminous efficacy of the flux) $\eta_{R}(\mathrm{~lm} / \mathrm{W})$ as defined in (6) and illustrated in the right-hand block in Figure 1.

\section{Mixtures of Narrowband Sources}

With the advent of high-output LED light sources, there is now the prospect of synthesizing white-light sources having spectral outputs confined to several rather narrow discrete bands within the visible range. It is therefore possible to avoid all radiation outside the visible range, as well as to attempt to select the wavelengths that produce the "best" visual effect in relation to any given lighting task.

The chief purpose of our research is therefore to define the optimum number and optimum wavelengths of such discrete bands-and so encourage the LED lighting industry to work towards the development of sources with the most appropriate spectra.

A further aim is to investigate the prospect of defining the optimum light-source spectrum, irrespective of the physical process of light production (whether LED or other alternative sources in the future) to simultaneously enhance both the efficacy and colour rendering performance of the source.

In the simulations and experimental work described below, a general aim was to attempt to find mixtures that would produce white light with CCTs in the range $2,800 \mathrm{~K}$ to $4,200 \mathrm{~K}$. This range covers the design requirements for a wide range of domestic, commercial, and office lighting applications.

4.1. Simulation of 3-Band Sources. An early computer-based simulation experiment [18] explored the trade-offs between colour rendering index and radiant luminous efficacy over a range of colour temperatures. Briefly, the computer program operated as follows.

The user provided an input colour temperature, and the program generated a reference SPD from Plank's formula at $5 \mathrm{~nm}$ intervals over the range $380-770 \mathrm{~nm}$. The chromaticity of the reference source was calculated, as well as those of the eight CIE test colour samples under the reference source. (By definition, this source would have $R_{a}=100$.) Also, the radiant luminous efficacy $\eta_{R}$ of the source was calculated. This source now became the "development source" which was gradually modified in the course of the program execution.

For the first $5 \mathrm{~nm}$ ( $380 \mathrm{~nm}$ centre wavelength) waveband of the "test source", the power was reduced by $20 \%$. The $R_{a}$ value for this test source was calculated and stored; the power in the $380 \mathrm{~nm}$ waveband was restored; the power in the next waveband $(385 \mathrm{~nm})$ was reduced. $R_{a}$ was calculated, and this was repeated for every waveband to give a file of $79 R_{a}$ values. The minimum $R_{a}$ was found. The waveband at which this occurred was the waveband for which a reduction of power produced the greatest loss of $R_{a}$. Conversely, the highest value indicated the waveband at which power can be reduced with minimum loss of $R_{a}$.

The "development source" SPD was then modified, waveband by waveband, by reducing the power proportionally by between zero (for the waveband of minimum $R_{a}$ ) and a maximum of $1 \%$ (for a waveband in which $R_{a}=100$ ). The $R_{a}$ and $\eta_{R}$ values were calculated, and the SPD was saved in a result file.

By the use of iteration, together with a results sorting process, it was possible to obtain different SPD shapes for spectra producing different combinations of $T_{C}, R_{a}$, and $\eta_{R}$. Figure 2 shows an example of a spectrum obtained in this way, having peak wavelengths at approximately 455,540 , and $620 \mathrm{~nm}$. This simulation experiment proved that it is possible to envisage a 3-band light source with both high $R_{a}$ and high $\eta_{R}$, provided that one is able to select the centre wavelengths and the bandwidths of the spectral bands. 


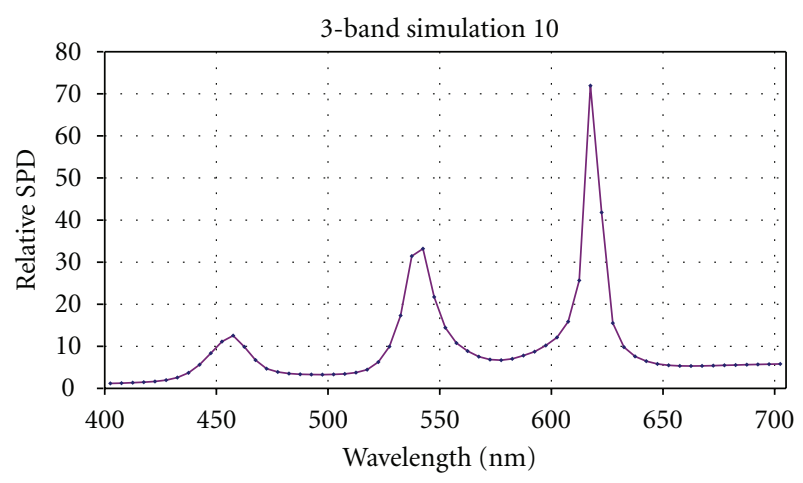

Figure 2: Example of a 3-band source simulation [18]. $T_{C}=$ 3232 K; CRI: $R_{a}=85.0 ; R_{b}=74.1 ; R_{c}=80.3, \eta_{R}=340$ lumens per radiant watt.

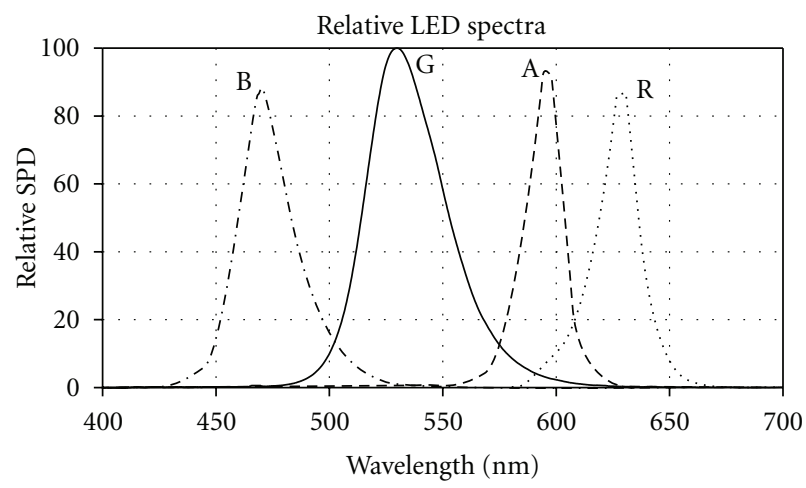

Figure 3: Measured SPDs of the four experimental LEDs Data normalized to the Green peak. B-blue, G-green, A-amber, and Rred Peak. Wavelengths: B $-470 \mathrm{~nm}, \mathrm{G}-530 \mathrm{~nm}, \mathrm{~A}-596 \mathrm{~nm}$, and R $-629 \mathrm{~nm}$.

To put Figure 2 results into context, a typical tungstenhalogen lamp operating at $3000 \mathrm{~K}$ has $R_{a} \approx 100$ and $\eta_{R} \approx$ $165 \mathrm{~lm} / \mathrm{W}$ (radiant).

4.2. Mixed-LED Sources. With LEDs it is possible to produce white-light mixtures of any selected correlated colour temperature, with any number of LEDs from two upwards, as long as devices are available that can produce the required wavelengths. Two-band mixtures will be the subject of future investigations. The present work focuses on mixtures utilizing 3 or more bands.

4.3. Mixtures of Real LEDs. A set of four LED sources (having spectra as shown in Figure 3) was used to carry out practical LED mixing experiments to confirm that such mixtures can perform effectively as white-light sources [19].

The LEDs and their control circuits were a package supplied by Philips Lighting, comprising one Xitanium LED electronic driver $(1.75 \mathrm{~A}, 24 \mathrm{~V})$ and four Philips LMS $2 \times 2$ (4 W, 20-24 V) LED lamps, each equipped with its individual Philips CI DALI/DMX controller (36W, 20-24V). The lamps were of four separate colours, Red, Amber, Green, Blue; each lamp comprised four LEDs. These were used in conjunction

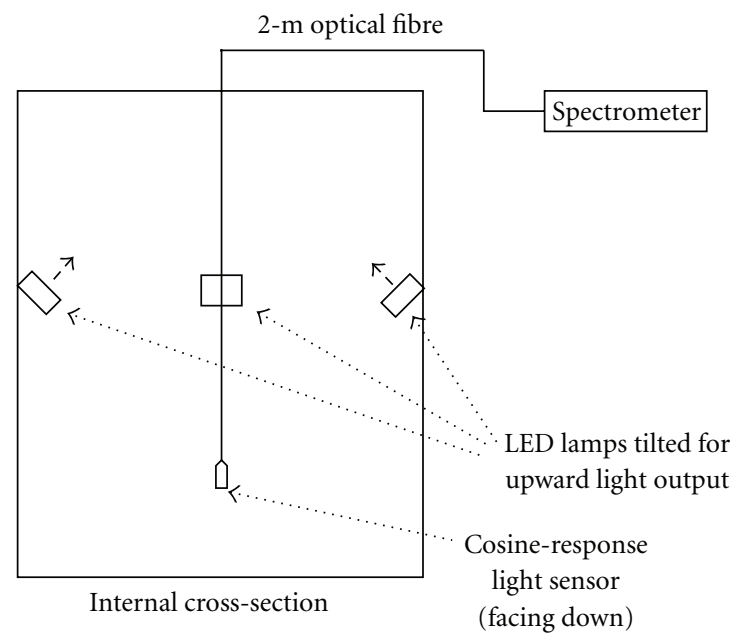

(a)

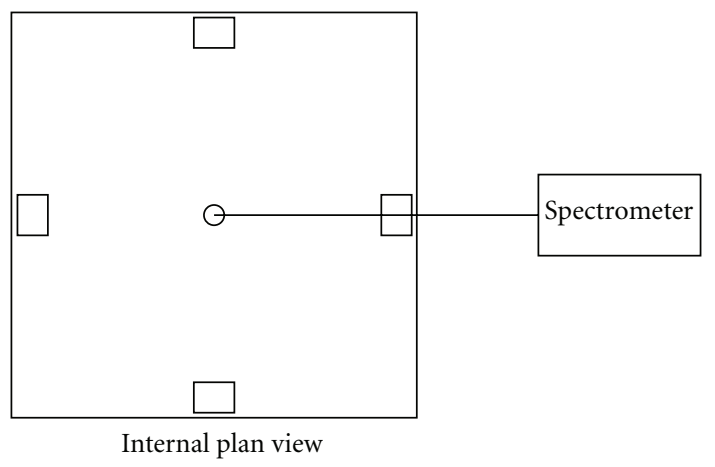

(b)

FIGURE 4: Schematic diagram of integrating enclosure (not to scale). See text for details.

with a Strand Lighting Accent DMX control station which permitted each lamp to be dimmed from maximum to zero output.

With this equipment it was possible to set up light mixtures of practically any colour within the gamut of the four primary lamp colours. Mixing experiments [19] were conducted using a rectangular integrating enclosure of approximately 0.5 metre side, shown schematically in Figure 4, and coated internally with a spectrally uniform matte white finish. The light sources were aimed upwards, and all measurements and observations were performed at the "floor level" inside the box, where the light mixing was checked and shown to be uniform. Spectrum measurements were taken using an Ocean Optics HR2000 spectrometer, calibrated in spectral radiance mode. CRI values and radiant efficacies were computed from the measured spectra.

Initial experiments focussed on 3-band mixtures (Red, Green, and Blue) since, as a general rule, the mixing adjustments are simpler when fewer sources are involved. Figure 5 is an example of such a 3-band mixture, with peak wavelengths at 470,530 , and $629 \mathrm{~nm}$.

The poor colour rendering obtained with this spectrum was surprising in view of its superficial similarity to the 


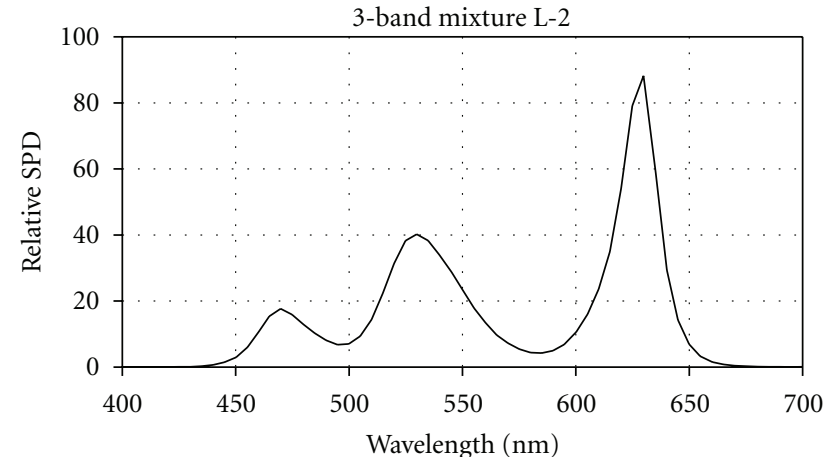

Figure 5: Relative SPD of 3-band LED mixture [19]. $T_{C}=3583 \mathrm{~K}$; CRI: $R_{a}=58.3 ; R_{b}=48.7 ; R_{c}=54.2, \eta_{R}=342$ lumens per radiant watt.

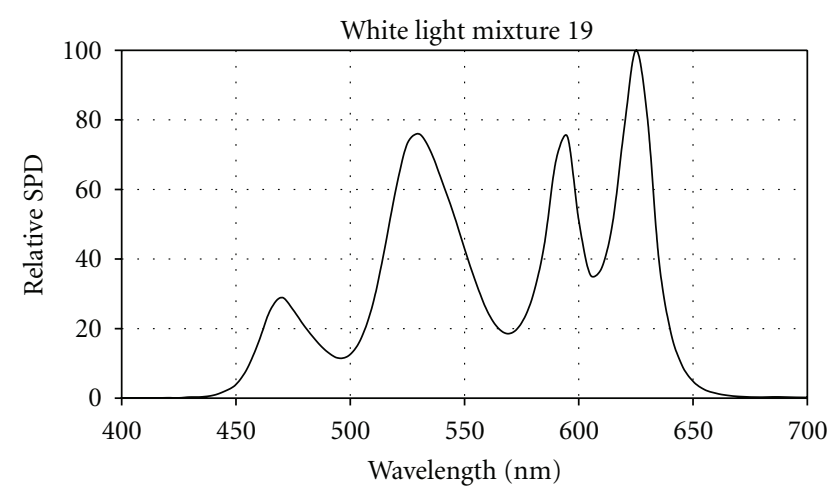

Figure 6: A 4-band LED mixture [19]. $T_{C}=3765 \mathrm{~K}$; CRI: $R_{a}=$ $86.7 ; R_{b}=71.5 ; R_{c}=80.2, \eta_{R}=406$ lumens per radiant watt.

spectrum of Figure 2. Comparisons show a fractional gain in radiant luminous efficacy, but about 33\% lower CRI values. The disparity of these results can be explained by the differences in the peak wavelengths and the bandwidths of the three bands, in the two instances.

In view of these results, and in common with other researchers in the field (e.g. [20]), it was decided to investigate the advantages of employing 4-band mixtures. A target was set to achieve a mixture with a colour temperature of approximately $3500 \mathrm{~K}$, together with the highest possible values for CRI and radiant luminous efficacy.

A number of practical mixing experiments were carried out, and an example of one of the better the results is given in Figure 6. This particular mixture gave one of the highest radiant luminous efficacy results $\left(\eta_{R}=406 \mathrm{~lm} / \mathrm{W}\right)$ achieved with this set of four LEDs. Subsequent simulations [21] proved that minor adjustments to the above mixture could improve CRI by about $10 \%-15 \%$, but at the expense of a $17 \%$ reduction in radiant luminous efficacy.

4.4. Visual Assessment of 4-Band LED Mixture. The above four LED sources were next used in a visual evaluation experiment [22] performed at the 2007 Museum Lighting Workshop in the USA. In this experiment, two viewing booths were set up back-to-back in a dimly lit room. Both

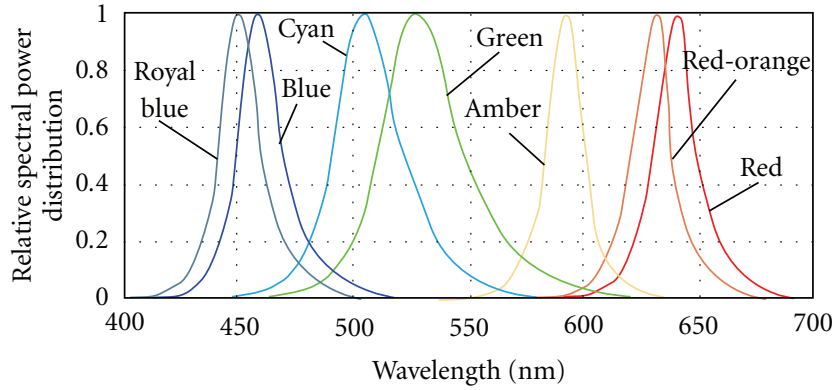

FIGURE 7: Relative SPDs for a family of seven LED sources. Source. Luxeon LED manual [23].

booths were illuminated at 50 lux The light source for Booth A was a tungsten halogen lamp, and for Booth $\mathrm{B}$, the outputs of the four LED sources were balanced to match the chromaticity of the illumination in Booth A. The light sources were hidden from the observers, and the lighting was constant throughout the survey.

A total of 114 subjects completed the survey in three sessions, with identical displayed objects in both of the booths for each session. The displayed objects were the following.

\section{Session 1: Gretagmacbeth ColorChecker.}

Session 2: Group of anthropological objects.

Session 3: Reproduction of a Claude Monet oil painting.

Subjects completed a questionnaire comprising four questions for each session.

The responses have been analysed, and the results summarized by Cuttle [22] as follows. "The responses suggest that subjects' ratings for clarity of detail [appeared to] be significantly higher for the LED lighting in Booth B... There is no indication of subjects discriminating against the appearance of the lighting, or the appearance of the displayed objects, due to the discontinuous spectrum of the LED combination".

Hence LED mixtures are capable of providing, not only high efficacies, but also very satisfactory colour rendering as judged by a group of highly discriminating museum experts.

4.5. Simulations of $n$-Band LED Sources. Recalling that the earlier computer modelling (Section 4.1) was not based on LEDs, it was decided that we should undertake further simulation work to determine whether the results of the practical mixing experiments (Sections 4.3, 4.4) could be further improved upon. The simulations and experiments performed with 3- and 4-band sources led to the concept of the $n$-band source, where $n$ is any suitable number greater than 2. Investigations into multiband sources were carried out by means of simulations based on one manufacturer's family of seven coloured LEDs [23] whose published spectra are depicted in Figure 7. 
TABLE 2: Comparison of predicted performance with $n \geq 4$ [24]: LED spectra as depicted in Figure 7.

\begin{tabular}{lcccc}
\hline & 4-band & 5-band & 6-band & 7-band \\
\hline$R_{a}$ & 91 & 94 & 94 & 92 \\
$R_{b}$ & 83 & 86 & 84 & 84 \\
$R_{c}$ & 88 & 91 & 90 & 89 \\
$\eta_{R}(\mathrm{~lm} / \mathrm{W})$ & 316 & 347 & 350 & 363 \\
$T_{C}(\mathrm{~K})$ & 3519 & 3467 & 3478 & 3492 \\
\hline
\end{tabular}

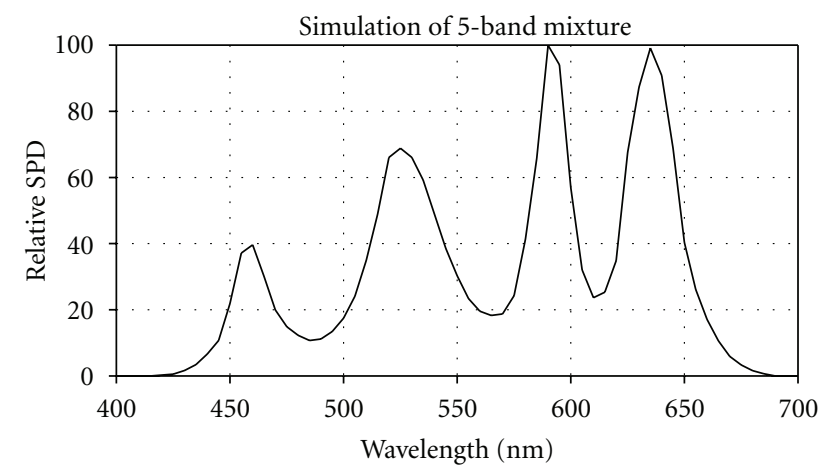

FIGURE 8: Relative SPD of the simulated 5-Band Mixture [24]. Note that the Red-Orange and Red bands have merged to form a single, broadened Red band. $T_{C}=3467 \mathrm{~K}$; CRI: $R_{a}=94 ; R_{b}=86 ; R_{c}=91$, $\eta_{R}=347$ lumens per radiant watt.

The spectral data for these sources were digitized to enable a series of simulations to be carried out [24] to investigate whether a larger number of sources in an $n$ band mixture can improve the performance according to the defined criteria. The optimization process was carried out using an operator-guided heuristic approach, in which the success of each trial mixture was judged by the ability to maximise both CRI and radiant efficacy, while also satisfying a colour temperature criterion.

The results of this investigation are given in Table 2 which summarizes the performance achieved with mixtures optimized for $R_{a}$ and $R_{b}$ with $T_{C} \cong 3500 \mathrm{~K}$, and for $n=4$ to $n=7$. It can be seen that the changes in the CRI figures for $n>4$ are marginal although the radiant luminous efficacies appear to increase with the number of bands used. The 7-band source appears to give an improvement in radiant luminous efficacy of approximately $15 \%$ over the 4-band source. However, this improvement may be difficult to achieve in practice because of the greater complexity in setting up and maintaining the correct balance of the sources in the 7-band mixture.

Taking all practical design criteria into consideration, one could make a case for selecting the 5-band source as the optimum - with its excellent combination of properties and moderately simple set-up procedures. The spectrum for this source is illustrated in Figure 8. The primaries used in this mixture are Blue, Green, Amber, Red-Orange, and Red. Because of the overlap of the Red-Orange and Red component spectra, this gives the appearance of a 4-band spectrum with a broadened Red band.
These simulations hold out the prospect of being able to achieve mixed-LED light sources with a highly satisfactory combination of efficacies and colour properties. It has to be recognized, however, that the calculations were all based on "typical" LED data-with no guarantee that a given set of real LEDs will exactly match these characteristics. For instance, the Luxeon data [23] indicates tolerances ranging from $\lambda_{\mathrm{P}} \pm 3.5 \mathrm{~nm}$ for the Red-Orange LED up to $\lambda_{\mathrm{P}}(+20 /-$ 10) $\mathrm{nm}$ for the Blue and Green LEDs (where $\lambda_{\mathrm{p}}$ signifies the peak wavelength for the specified LED colour). In addition, most manufacturers indicate temperature dependencies of both lumen output and $\lambda_{\mathrm{P}}$.

Changes such as those mentioned here would clearly have a significant impact on the results for $T_{C}, C R I$ and $\eta_{R}$, and these effects will form part of the next phase of our investigations. The extent of the changes will depend on the magnitudes of the wavelength deviations for each colour in the mixture. To the best of our knowledge, no literature currently exists on the effects of spectral tolerances in $n$-band LED mixtures. As a gauge of a "worst-case" estimate, one may refer back to the comparisons of the results for Figures 2 and 5 in Section 4.3. In terms of experimental data using actual LEDs, Chhajed et al. [25] have investigated the effect of junction temperature on the $R_{a}$ of a three-LED mixture at approximately $6500 \mathrm{~K}\left(T_{C}\right)$. They showed that, for an increase from $20^{\circ} \mathrm{C}$ to $80^{\circ} \mathrm{C}$ junction temperature, $R_{a}$ falls from 84 to 72 . They also show that a rebalancing of the mixture at the higher temperature can restore the $R_{a}$ to 81 . Thus, provided that one can adequately control the balance of a 3-LED mixture, the temperatureinduced spectrum shifts for this temperature range will apparently still be responsible for a loss of $\geq 3$ units of $\mathrm{Ra}$.

\section{Intelligent Spectral Design}

Because the process followed in Section 4.5 was a heuristic search for an optimal $n$-band LED mixture, there was no guarantee that the results obtained were necessarily the 'best' solutions. We have therefore embarked on a process of "intelligent spectral design" in an attempt to maximise the search space. The intention is to identify a wider range of potential solutions, with a higher likelihood of finding a true optimum. Such processes include evolutionary optimization [26], genetic algorithms [27], and differential evolution (DE) [28].

To this end, a series of investigations has commenced into the use of artificial intelligence techniques as an aid to the search for an engineering optimum. Some preliminary results indicate promising trends and are quoted in a recent conference paper [15]. In addition to optimizing for $R_{a}, R_{b}, R_{c}$, and $\eta_{R}$, the process here included the optimization of $R_{\min }$, the lowest $R_{i}$ value in the test, and identification of $i_{\min }$, the sample number that gave this $R_{\min }$ result. This investigation worked with the same set of seven LEDs as described in Section 4.5, and it yielded very similar general trends. An indicative spectrum is shown in Figure 9, together with a summary of significant results in Table 3 . 
TABLE 3: Comparison of DE-optimised mixtures with $n \geq 5$ : LED spectra as depicted in Figure 7.

\begin{tabular}{lccc}
\hline$n$ & 5 & 5 & 6 \\
\hline$R_{a}$ & 95 & 96 & 95 \\
$R_{b}$ & 85 & 86 & 86 \\
$R_{c}$ & 91 & 91 & 91 \\
$\eta_{R}(\mathrm{~lm} / \mathrm{W})$ & 343 & 338 & 334 \\
$T_{C}(\mathrm{~K})$ & 3269 & 3356 & 3541 \\
\hline
\end{tabular}

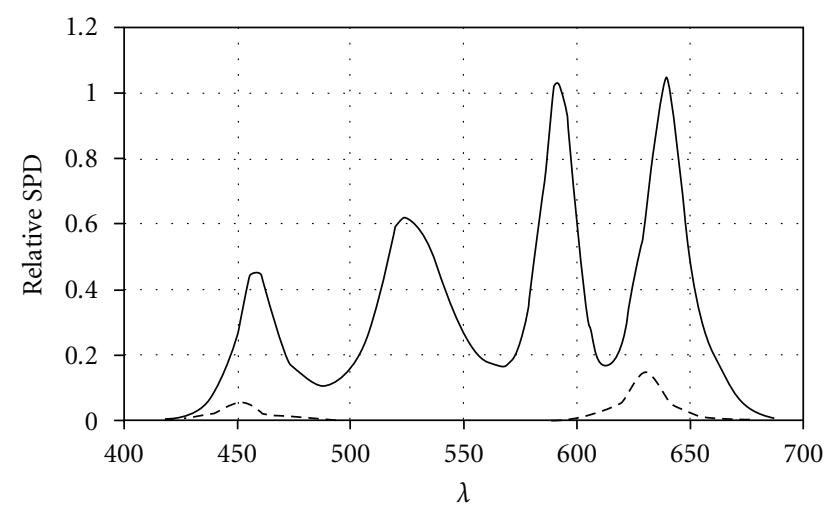

FIGURE 9: Relative SPD of a simulated 6-Band Mixture (solid line) [15]. Note that the two dashed spectral peaks represent the (minor) contributions to the mixture made by the Royal-Blue and RedOrange LEDs. $T_{C}=3541 \mathrm{~K}$; CRI: $R_{a}=95 ; R_{b}=86 ; R_{c}=91$; $R_{\min }=75 ; i_{\min }=12, \eta_{R}=334$ lumens per radiant watt.

However, because this work is in its early stages and is ongoing, it would be premature to draw any firm conclusions at this time. The next phase of this work is planned to include investigations of the effects of spreads in the peak wavelength values, as well as temperature coefficient effects.

\section{Visualization of Colour Rendering}

Because the majority of the simulation results (Sections 4.1, 4.5 and 5) have not been implemented as "real" mixtures, there exists a need to be able to display the effects of different SPDs to convince nontechnical users of the benefits of the mixtures that have been identified as potential optima. To this end, work has been undertaken to design a visualization tool $[13,14,29]$ to provide this facility. This has already proved its worth and will be developed further as an adjunct to the work on intelligent spectral design.

Figure 10 provides an illustration of the versatility of this approach, allowing for the comparison of real measured spectra with synthetic or simulated spectra. The colours are computed from the knowledge of the source SPDs (real or synthetic) together with the defined reflectance spectra for the 14 CIE test colours [5]. The system is intended for display on a computer screen, and it includes operator-controlled settings for colour management $[13,14]$.

Two further approaches are being developed for the psychophysical evaluation of narrow-band mixtures. (a) Further colour mixing experiments with real LEDs, to extend the work described in Section 4.4.

(b) The design of a highly flexible light source with fully selectable spectral content. A prototype version, known as the "Spectra" illuminator, has previously been constructed [30] and is under further development (to be the subject of a future paper).

\section{Conclusions}

Practical experimentation has proved that it is possible to create highly effective 4-band LED mixtures, in terms of the criteria defined earlier in this paper. It has also been demonstrated by simulation that further (minor) performance improvements are possible by the introduction of appropriate additional bands into the mixture.

Attention is drawn particularly to the following.

(i) The work reported here has been at the level of pilot studies to investigate the feasibility of using mixtures of narrow-band LEDs as white light sources possessing optimal combinations of radiant efficacy and colour rendering performance.

(ii) The performance of any given mixture is governed by the peak wavelength and the bandwidth of each of the bands used, as well as the numbers of bands, and possibly also the shape of the power distribution function within each band.

(iii) Future investigations will determine the effects of spreads in the LED characteristics, particularly the sensitivity of the results to possible changes in the peak wavelengths and the bandwidths radiated by the LEDs.

(iv) It is recognized that there is a need to implement further mixtures of real narrow-band sources in order to validate the computer modelling results already achieved as well as those contemplated for the future.

(v) Further subjective validation will be required to convince potential users of the utility of sources of the types envisaged here. Such testing can initially be carried out in laboratory settings, but should also be applied to real visual tasks in "real life" situations.

\section{Acknowledgments}

The authors wish to acknowledge the advice, assistance, and material support that has been provided by the Institute for Biomedical Technologies at Auckland University of Technology, the Getty Conservation Institute Los Angeles, Philips Lighting New Zealand, Professional Lighting Services Ltd Auckland, and Virtualight Ltd Auckland. In particular, they wish to thank Christopher (Kit) Cuttle for providing the original impetus for this work, and for continuing to provide advice and support for their research group. The current phase of the work is supported by the RDTT Fund of the Manukau Institute of Technology and by the EET School at the Manukau Institute of Technology. 


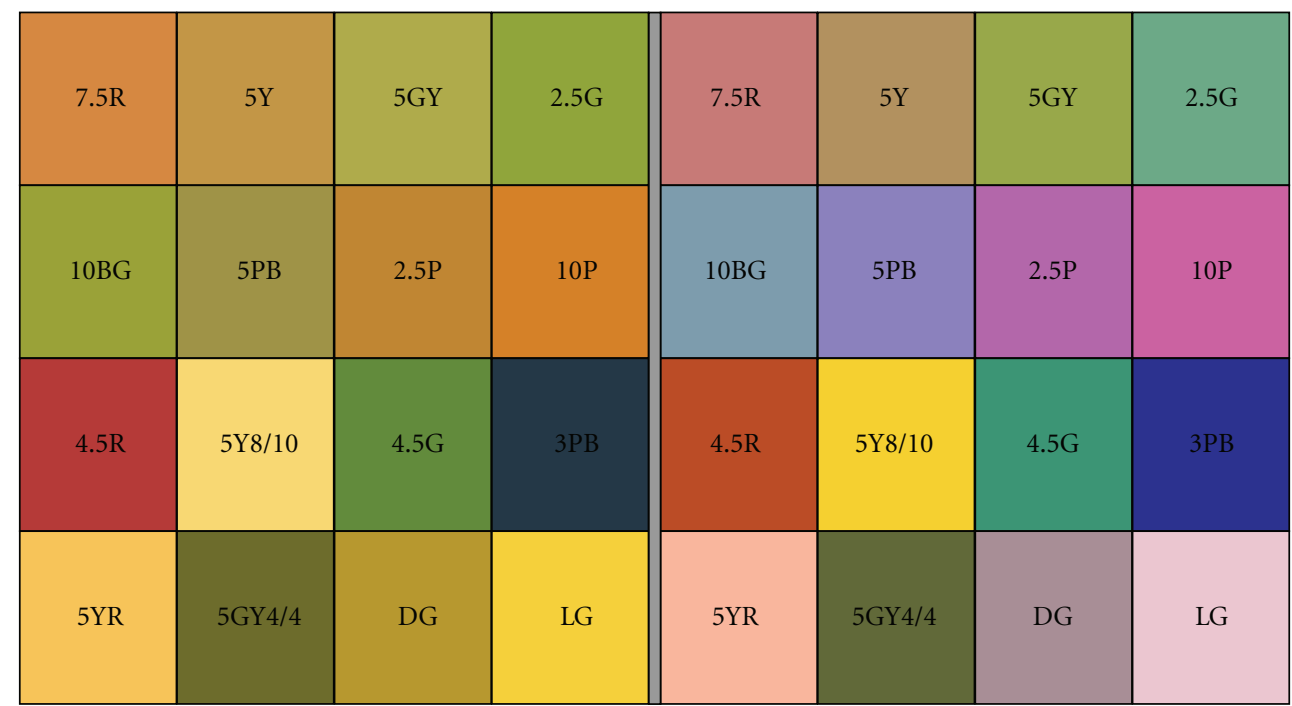

FIGURE 10: Visualization of the comparison of the colour rendering of two sources [29] Left: a measured white LED spectrum ( $\left.T_{C} \approx 3000 \mathrm{~K}\right)$. Right: the synthetic equi-energy spectrum $\left(T_{C} \approx 5450 \mathrm{~K}\right)$. Note that each half of the display shows the 14 CIE test colours [5] plus two synthetic grey colours (DG, LG) at bottom-right of each set.

\section{References}

[1] M. A. Cayless and A. M. Marsden, Eds., Lamps and Lighting, Edward Arnold, London, UK, 3rd edition, 1983, pp. 344-358.

[2] M. Harris, "Let there be light," IET Engineering \& Technology, vol. 4, no. 20, pp. 18-21, 2009.

[3] D. B. Judd and G. Wyszecki, Color in Business, Science and Industry, John Wiley \& Sons, New York, NY, USA, 3rd edition, 1975.

[4] G. E. Lighting, "Spectrum," (Lamp product catalogue 2009/2010).

[5] Commission Internationale de l'Eclairage, "Method of measuring and specifying colour rendering properties of light sources," CIE Technical Report 13.3, CIE Central Bureau, Vienna, Austria, 1995.

[6] N. Sándor and J. Schanda, "Visual colour rendering based on colour difference evaluations," Lighting Research and Technology, vol. 38, no. 3, pp. 225-239, 2006.

[7] Commission Internationale de l'Eclairage, "Colour rendering of white LED light sources," CIE Technical Report 177, CIE Central Bureau, Vienna, Austria, 2007.

[8] Commission Internationale de l'Eclairage, "Colorimetry," CIE Technical Report 15, CIE Central Bureau, Vienna, Austria, 2004.

[9] M. A. Cayless and A. M. Marsden, Eds., Lamps and Lighting, Edward Arnold, London, UK, 3rd edition, 1983, pp. 53-55.

[10] G. Wyszecki and W. S. Stiles, Color Science, John Wiley \& Sons, New York, NY, USA, 2nd edition, 1982, pp. 431-432.

[11] D. B. Judd and G. Wyszecki, Color in Business, Science and Industry, John Wiley \& Sons, New York, NY, USA, 3rd edition, 1975.

[12] G. Wyszecki and W. S. Stiles, Color Science, John Wiley \& Sons, New York, NY, USA, 2nd edition, 1982, p. 828.

[13] A. N. Chalmers, L. Wang, and M. V. Ramos, "A virtual imaging system for colour research," in Proceedings of the 23rd International Conference on Image and Vision Computing New Zealand (IVCNZ'08), pp. 1-6, November 2008.
[14] L. Wang, A. N. Chalmers, and M. V. Ramos, "A research tool for colour rendering," in Proceedings of the 15th Electronics New Zealand Conference (ENZCon '08), pp. 1-6, Auckland, New Zealand, November 2008.

[15] S. Soltic and A. N. Chalmers, "Differential evolution and its application to intelligent spectral design," in Proceedings of the 16th Electronics New Zealand Conference (ENZCon '09), pp. 19-24, Dunedin, New Zealand, November 2009.

[16] C. Cuttle, "New opportunities for LEDs in museum display lighting," in Proceedings of the Professional Lighting Design Convention, pp. 38-44, Berlin, Germany, October 2009.

[17] G. Wyszecki and W. S. Stiles, Color Science, New York, NY, USA, John Wiley \& Sons, 2nd edition, 1982, pp. 256-259.

[18] C. Cuttle, "Spectra project: stage one," Report, Getty Conservation Institute, Auckland, New Zealand, 2004.

[19] P. Luong and A. N. Chalmers, "White light mixture using 4 LEDs," Project Report, Manukau Institute of Technology, 2006.

[20] F. Viénot, E. Mahler, L. Serreault, et al., "Discriminating colours under LED illumination," in Proceedings of the 10th Congress of the International Colour Association (AIC Colour '05), pp. 33-36, 2005.

[21] C. Cuttle, "LED Lighting project," Report for Getty Conservation Institute, 2006.

[22] C. Cuttle, “Assessment: LED versus MR-16," Report for Getty Conservation Institute, 2007.

[23] Lumileds Lighting, LUXEON ${ }^{\circledR}$ K2 Emitter, Technical Datasheet DS51, Lumileds Lighting U.S., LLC, San Jose, Calif, USA, 2006.

[24] L. Wang and A. N. Chalmers, High Luminous Efficacy Light Sources, BE(Hons), MiniConference, Manukau Institute of Technology, 2007.

[25] S. Chhajed, Y. Xi, Y.-L. Li, Th. Gessmann, and E. F. Schubert, "Influence of junction temperature on chromaticity and colorrendering properties of trichromatic white-light sources based on light-emitting diodes," Journal of Applied Physics, vol. 97, no. 5, Article ID 054506, 8 pages, 2005.

[26] K.-S. Sohn, D. H. Park, S. H. Cho, J. S. Kwak, and J. S. Kim, "Computational evolutionary optimization of red phosphor 
for use in tricolor white LEDs," Chemistry of Materials, vol. 18, no. 7, pp. 1768-1772, 2006.

[27] N. H. Eklund and M. J. Embrechts, "Multi-objective optimization of spectra using genetic algorithms," Journal of the Illuminating Engineering Society, vol. 30, no. 2, pp. 65-71, 2001.

[28] R. Storn and K. Price, "Differential evolution: a simple and efficient heuristic for global optimization over continuous spaces," Journal of Global Optimization, vol. 11, no. 4, pp. 341359, 1997.

[29] A. N. Chalmers, L. Wang, and S. Soltic, "A colour visualization tool for light-source research," in Proceedings of the 11th Congress International Colour Association (AIC Colour'09), pp. 1-7, Sydney, Australia, 2009.

[30] A. N. Chalmers, C. Cuttle, and R. Van Ryn, "An experimental illuminator for museum conservation lighting," in Australian Conference on Optical Fibre Technology/Australian Optical Society (ACOFT/AOS '06), Melbourne, Australia, July 2006. 

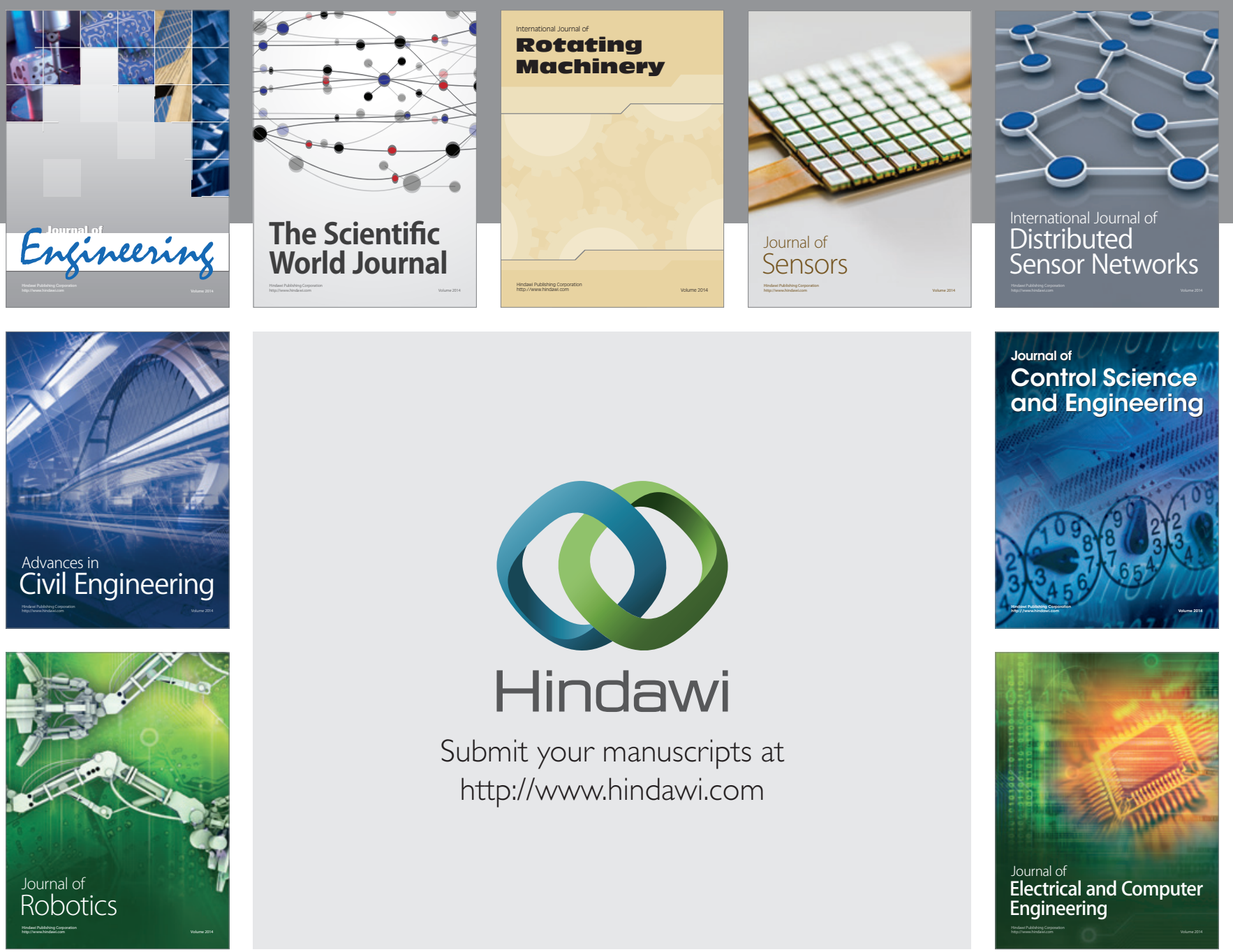

Submit your manuscripts at

http://www.hindawi.com
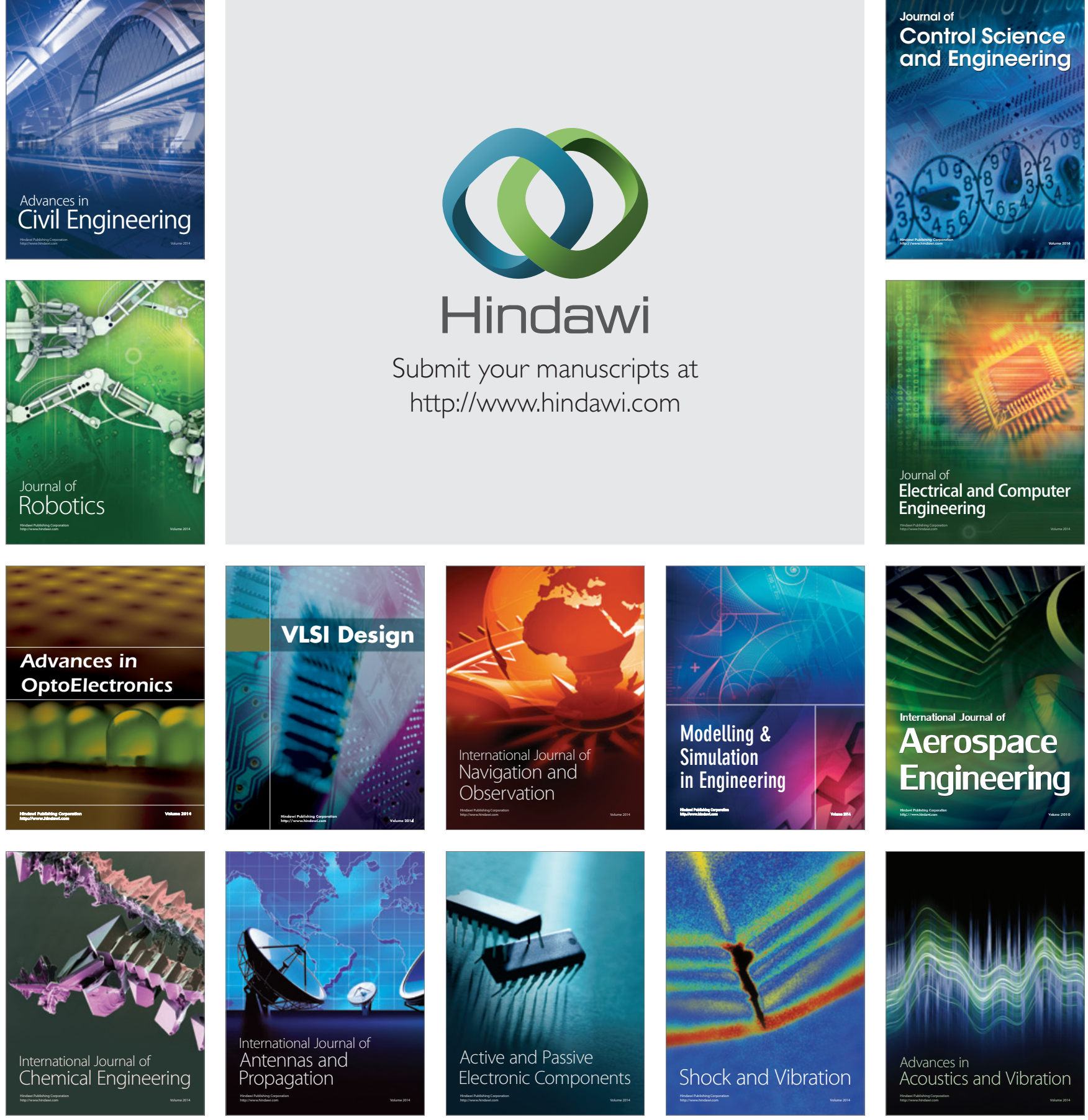